\title{
How many subjects? Statistical power analysis in research
}

\author{
STEPHEN DUBIN \\ Drexel University, Philadelphia, Pennsylvania
}

\section{How many subjects? Statistical power analysis in research}

By Helena Chmura Kraemer \& Sue Thiemann. 1987, Newbury Park, CA: Sage Publications, Inc. 120 pp. ISBN: 0-8039-2944-8, \$17.95.

It is most satisfying to find a statistical book that is informative and mathematically rigorous, yet very pleasant reading in a manageable format. How Many Subjects?, by Helena Chmura Kraemer and Sue Thiemann, is just such a little gem. This stylishly slim volume (120 pages, of which 20 are chapter dividers or blanks) presents the authors' method of sample size estimation for a wide variety of statistical procedures, along with much useful instruction and many insights into general experimental design.

Research investigators are increasingly being called upon to justify the number of observations required for their work (cf. the NIH Guide for the Care and Use of Laboratory Animals, Appendix D). In addition to obviating unnecessary distress to subjects, careful planning of experiments promotes economy and plain good science. Of the many sources of information on statistically appropriate sample size, many are task-specific, being limited to a single or small number of statistical procedures. Some are exhaustive and exhausting because of the mass and diversity of material. A regrettable few-unfortunately attractive to the beginner because of their simplicity-handle only Type I $(\alpha)$ error and ignore the very important power side (Type II or $1-\beta$ ) of the sample size issue.

Kraemer and Thiemann's book begins with a brief but lucid overview of the concepts of statistical hypothesis testing, critical effect size, and power analysis in the overall context of research study planning. A very elegant feature of the book is the authors' innovation of a single method of power calculation that is appropriate for a wide variety of different tests. Not only is this aesthetically

Stephen Dubin, VMD, PhD, a "recovering veterinarian," is clinical professor of biomedical engineering and science at Drexel University in Philadelphia. His address is: Drexel University, 32nd \& Chestnut Sts., Philadelphia, PA 19104. appealing, but it also mitigates the effort of unraveling the mathematical background. The authors develop the basis of their method with concise mathematical rigor. In other words, the serious student should be prepared to read the references. They then apply the method to a wide variety of statistical procedures, including $t$ tests, analysis of variance, various forms of correlation and regression analysis, and nonparametric (binomial) test procedures. Application of the method to contingency table (chi-square) testing is possible but a bit more complicated than it is for the other statistical tests. The final part of the book contains a summary table of the formulas and the master tables.

Typically, one calculates a value of $\delta$, which is in turn a parameter for the calculation of $\Delta$. The reader then uses the master tables according to the required levels of alpha and power and the value of $\Delta$ for their proposed study.

Rather than provide a wide variety of examples, Kraemer and Thiemann present a single "threaded" example pertaining to coffee drinking and the Cornell Medical Index (CMI). They proceed to illustrate the estimation of appropriate sample size for each of the statistical procedures with reference to various ways in which the same coffee/CMI study might be structured and analyzed. This approach certainly disqualifies the book as a "cookbook" in the manner of Schaum's Outlines. It does, however, teach much more about experimental design than just sample size.

Finding a sample size using this book requires several steps of computation-sometimes involving transcendental functions-and then interpolation within the master table. This becomes tedious and possibly vulnerable to error when the reader wishes to ask multiple "what if" questions about an experimental design. How Many Subjects? is very concise; the nonexpert reader will have to read it through with careful attention. Everything is there, but only in one place!

This book is an excellent choice for the research investigator who wants a pleasant but rigorous introduction to power analysis. It is perhaps the sole source for taking students beyond the cookbook stage of sample size determination. 